\title{
Convergence theorems of a new iteration for asymptotically nonexpansive mappings in Banach spaces
}

\author{
Jong Kyu Kim* and Won Hee Lim
}

\section{"Correspondence:}

jongkyuk@kyungnam.ac.kr Department of Mathematics Education, Kyungnam University, Changwon, Gyeongnam 631-701 Korea

\begin{abstract}
In this paper, the problem of modified iterative approximation of common fixed points of asymptotically nonexpansive is investigated in the framework of Banach spaces. Weak convergence theorems are established.

MSC: $47 \mathrm{H} 09 ; 47 \mathrm{~J} 05 ; 47 \mathrm{~J} 25 ; 47 \mathrm{H} 25$
\end{abstract}

Keywords: asymptotically nonexpansive mapping; common fixed point; implicit Ishikawa-type iterative process

\section{Introduction}

Fixed-point theory as an important branch of nonlinear analysis has been applied in the study of nonlinear phenomena. The theory itself is a beautiful mixture of analysis, topology, and geometry. Recently, iterative algorithms for finding common fixed points of nonlinear mappings have been considered by many authors. The well-known convex feasibility problem capture application in various disciplines such as image restorations, and radiation therapy treatment planning is to find a point in the intersection of common fixed-point sets of nonlinear mappings (see, [1-6]).

From the method of generating iterative sequence, we can divide iterative algorithms into explicit algorithms. Recently, both explicit Mann iterative algorithms and implicit Mann-iterative algorithms have been extensively studied for approximating common fixed points of nonlinear mappings (see [7-17]).

In this paper, we consider the problem of approximating a common fixed point of asymptotically nonexpansive mappings based on a general implicit iterative algorithm, which includes an explicit process as a special case. The organization of this paper is as follows. In Section 2, we provide some necessary preliminaries. In Section 3, weak convergence theorems are established in a uniformly convex Banach space.

\section{Preliminaries}

Let $E$ be a real Banach space. $E$ is said to be uniformly convex if for any two sequences $\left\{x_{n}\right\}$ and $\left\{y_{n}\right\}$ in $E$ such that $\left\|x_{n}\right\|=\left\|y_{n}\right\|=1$ and $\lim _{n \rightarrow \infty}\left\|x_{n}+y_{n}\right\|=2$, then $\lim _{n \rightarrow \infty}\left\|x_{n}-y_{n}\right\|=0$ holds. It is known that a uniformly convex Banach space is reflexive.

In this paper, we use the symbols $\rightarrow$ and $\rightarrow$ denote weak convergence and strong convergence, respectively. $E$ is said to have Opial's condition (see [18]) if, for each sequence 
$\left\{x_{n}\right\}$ in $E, x_{n} \rightarrow x$ implies that

$$
\liminf _{n \rightarrow \infty}\left\|x_{n}-x\right\|<\liminf _{n \rightarrow \infty}\left\|x_{n}-y\right\|, \quad \forall y \in E(y \neq x) .
$$

Let $C$ be a nonempty subset of $E$, and $T: C \rightarrow C$ a mapping. In this paper, the symbol $F(T)$ stands for the fixed point set of $T$. $T$ is said to be nonexpansive if

$$
\|T x-T y\| \leq\|x-y\|, \quad \forall x, y \in C
$$

$T$ is said to be asymptotically nonexpansive if there exists a sequence $\left\{k_{n}\right\} \subset[1, \infty)$ with $k_{n} \rightarrow 1$ as $n \rightarrow \infty$ such that

$$
\left\|T^{n} x-T^{n} y\right\| \leq k_{n}\|x-y\|, \quad \forall x, y \in C, \forall n \geq 1
$$

The class of asymptotically nonexpansive mappings was introduced by Goebel and Kirk [19] as a generalization of the class of nonexpansive mappings. They proved that if $C$ is a nonempty, closed, convex, and bounded subset of a real uniformly convex Banach space, then every asymptotically nonexpansive self mapping has a fixed point (see [19]).

In order to prove our main results, we still need the following lemmas.

Lemma 2.1 [20] Let $C$ be a nonempty, closed, and convex subset of a uniformly convex Banach space E. Let $T: C \rightarrow C$ be an asymptotically nonexpansive mapping. Then $I-T$ is demiclosed at zero, that is, $x_{n} \rightarrow x$ and $x_{n}-T x_{n} \rightarrow 0$ imply that $x=T x$.

Lemma $2.2[21]$ Let $\left\{a_{n}\right\},\left\{b_{n}\right\}$, and $\left\{c_{n}\right\}$ be three nonnegative sequences satisfying the following condition:

$$
a_{n+1} \leq\left(1+b_{n}\right) a_{n}+c_{n}, \quad \forall n \geq n_{0}
$$

where $n_{0}$ is some nonnegative integer, $\sum_{n=1}^{\infty} b_{n}<\infty$ and $\sum_{n=1}^{\infty} c_{n}<\infty$. Then the $\lim _{n \rightarrow \infty} a_{n}$ exists.

Lemma 2.3 [15] Let E be a uniformly convex Banach space, $r>0$ a positive number and $B_{r}(0)$ a closed ball of $E$ with the center at zero. Then there exists a continuous, strictly increasing, and convex function $g:[0, \infty) \rightarrow[0, \infty)$ with $g(0)=0$ such that

$$
\left\|\sum_{s=1}^{m}\left(\alpha_{s} x_{s}\right)\right\|^{2} \leq \sum_{s=1}^{m}\left(\alpha_{s}\left\|x_{s}\right\|^{2}\right)-\alpha_{i} \alpha_{j} g\left(\left\|x_{i}-x_{j}\right\|\right), \quad \forall i, j \in\{1,2, \ldots, r\}
$$

where $x_{1}, x_{2}, \ldots, x_{m} \in B_{r}(0)$, and $\alpha_{1}, \alpha_{2}, \ldots, \alpha_{m} \in(0,1)$ with $\sum_{i=1}^{m} \alpha_{i}=1$.

\section{Main Results}

Before starting the main results in this paper, we give the implicit iterative process first. Let $C$ be a nonempty, closed, and convex subset of a Banach space $E$. Let $T: C \rightarrow C$ be an asymptotically nonexpansive mapping with the sequence $\left\{k_{n}\right\}$. For every $u \in C$ and $t_{n} \in(0,1)$, Define a mapping $T_{n}: C \rightarrow C$ below

$$
T_{n}=t_{n} u+\left(1-t_{n}\right) T^{n} x, \quad \forall x \in C, \forall n \geq 1 .
$$


If $\left(1-t_{n}\right) k_{n}<1$, for every $n \geq 1$, then $T_{n}$ is a contraction. In the light of the Banach contraction principle, we see that there exists a unique fixed point of $T_{n}$, for every $n \geq 1$.

Let $x_{0}$ be chosen arbitrarily and $r \geq 1$ a positive integer. Let $\left\{\alpha_{n}\right\},\left\{\beta_{n, 1}\right\},\left\{\beta_{n, 2}\right\}, \ldots,\left\{\beta_{n, r}\right\}$, $\left\{\gamma_{n, 1}\right\},\left\{\gamma_{n, 2}\right\}, \ldots$, and $\left\{\gamma_{n, r}\right\},\left\{a_{n}\right\},\left\{b_{n, 1}\right\},\left\{b_{n, 2}\right\}, \ldots,\left\{b_{n, r}\right\},\left\{c_{n, 1}\right\},\left\{c_{n, 2}\right\}, \ldots$, and $\left\{c_{n, r}\right\}$ be real number sequences in $(0,1)$ such that

$$
\alpha_{n}+\sum_{m=1}^{r} \beta_{n, m}+\sum_{m=1}^{r} \gamma_{n, m}=a_{n}+\sum_{m=1}^{r} b_{n, m}+\sum_{m=1}^{r} c_{n, m}=1
$$

Let $S_{m}, T_{m}: C \rightarrow C$ be asymptotically nonexpansive mappings, for every $m \in\{1,2, \ldots, r\}$.

Find $x_{1}, y_{1}$ by solving the following equations:

$$
\begin{aligned}
& x_{1}=\alpha_{1} x_{0}+\sum_{m=1}^{r} \beta_{1, m} S_{m} x_{0}+\sum_{m=1}^{r} \gamma_{1, m} T_{m} y_{1}, \\
& y_{1}=a_{1} x_{1}+\sum_{m=1}^{r} b_{1, m} S_{m} x_{1}+\sum_{m=1}^{r} c_{1, m} T_{m} x_{1} .
\end{aligned}
$$

Find $x_{2}, y_{2}$ by solving the following equations:

$$
\begin{aligned}
& x_{2}=\alpha_{2} x_{1}+\sum_{m=1}^{r} \beta_{2, m} S_{m}^{2} x_{1}+\sum_{m=1}^{r} \gamma_{2, m} T_{m}^{2} y_{2}, \\
& y_{2}=a_{2} x_{2}+\sum_{m=1}^{r} b_{2, m} S_{m}^{2} x_{2}+\sum_{m=1}^{r} c_{2, m} T_{m}^{2} x_{2},
\end{aligned}
$$

Find $x_{n}, y_{n}$ by solving the following equations:

$$
\begin{aligned}
& x_{n}=\alpha_{n} x_{n-1}+\sum_{m=1}^{r} \beta_{n, m} S_{m}^{n} x_{n-1}+\sum_{m=1}^{r} \gamma_{n, m} T_{m}^{n} y_{n}, \\
& y_{n}=a_{n} x_{n}+\sum_{m=1}^{r} b_{n, m} S_{m}^{n} x_{n}+\sum_{m=1}^{r} c_{n, m} T_{m}^{n} x_{n} .
\end{aligned}
$$

In view of the above, we have the following implicit iterative algorithm:

$$
\left\{\begin{array}{l}
x_{0} \in C, \\
x_{n}=\alpha_{n} x_{n-1}+\sum_{m=1}^{r} \beta_{n, m} S_{m}^{n} x_{n-1}+\sum_{m=1}^{r} \gamma_{n, m} T_{m}^{n} y_{n}, \\
y_{n}=a_{n} x_{n}+\sum_{m=1}^{r} b_{n, m} S_{m}^{n} x_{n}+\sum_{m=1}^{r} c_{n, m} T_{m}^{n} x_{n}, \quad \forall n \geq 1 .
\end{array}\right.
$$

Now we show that $(\Upsilon)$ can be employed to approximate fixed points of asymptotically nonexpansive mapplings, which are assumed to be Lipschitz continuous. Let $S_{m}: C \rightarrow C$ be an asymptotically nonexpansive mapping with the sequence $\left\{s_{n, m}\right\}$, and $T_{m}: C \rightarrow C$ an asymptotically nonexpansive mapping with the sequence $\left\{t_{n, m}\right\}$, for every $m \in\{1,2, \ldots, r\}$, 
where $r \geq 1$ is some positive integer. Define a mapping $C_{n}: C \rightarrow C$ by

$$
\begin{aligned}
C_{n}(x)= & \alpha_{n} x_{n-1}+\sum_{m=1}^{r} \beta_{n, m} S_{m}^{n} x_{n-1} \\
& +\sum_{n}^{m} \gamma_{n, m} T_{m}^{n}\left(a_{n} x+\sum_{m=1}^{r} b_{n, m} S_{m}^{n} x+\sum_{m}^{n} c_{n, m} T_{m}^{n} x\right), \quad \forall n \geq 1 .
\end{aligned}
$$

It follows that

$$
\begin{aligned}
& \left\|C_{n}(x)-C_{n}(y)\right\| \\
& \leq \sum_{m=1}^{r} \gamma_{n, m} t_{n}\left\|a_{n}(x-y)+\sum_{m=1}^{r} b_{n, m}\left(S_{m}^{n} x-S_{m}^{n} y\right)+\sum_{m=1}^{r} c_{n, m}\left(T_{m}^{n} x-T_{m}^{n} y\right)\right\| \\
& \leq \sum_{m=1}^{r} \gamma_{n, m} t_{n}\left(a_{n}+\sum_{m=1}^{r} b_{n, m} s_{n}+\sum_{m=1}^{r} c_{n, m} t_{n}\right)\|x-y\|, \quad \forall x, y \in C,
\end{aligned}
$$

where $t_{n}=\max \left\{t_{n, m}: 1 \leq m \leq r\right\}$ and $s_{n}=\max \left\{s_{n, m}: 1 \leq m \leq r\right\}$.

If $\sum_{m=1}^{r} \gamma_{n, m} t_{n}\left(a_{n}+\sum_{m=1}^{r} b_{n, m} s_{n}+\sum_{m=1}^{r} c_{n, m} t_{n}\right)<1$ for all $1 \leq m \leq r, n \geq 1$, then $C_{n}$ is a contraction. Hence, by the Banach contraction principle, there exists a unique fixed point $x_{n} \in C$ such that

$$
\begin{aligned}
x_{n}= & C_{n}(x)=\alpha_{n} x_{n-1}+\sum_{m=1}^{r} \beta_{n, m} S_{m}^{n} x_{n-1} \\
& +\sum_{n}^{m} \gamma_{n, m} T_{m}^{n}\left(a_{n} x+\sum_{m=1}^{r} b_{n, m} S_{m}^{n} x+\sum_{m}^{n} c_{n, m} T_{m}^{n} x\right), \quad \forall n \geq 1 .
\end{aligned}
$$

That is, the implicit iterative algorithm $(\Upsilon)$ is well defined.

Now, we are in a position to give our main results.

Theorem 3.1 Let $C$ be a nonempty, closed, and convex subset of a uniformly convex Banach space E. Let $S_{m}: C \rightarrow C$ be an asymptotically nonexpansive mapping with the sequence $\left\{s_{n, m}\right\}$, and $T_{m}: C \rightarrow C$ an asymptotically nonexpansive mapping with the sequence $\left\{t_{n, m}\right\}$, for every $m \in\{1,2, \ldots, r\}$, where $r \geq 1$ is some positive integer. Assume that

$$
\mathcal{F}=\bigcap_{m=1}^{r} F\left(S_{m}\right) \cap \bigcap_{m=1}^{r} F\left(T_{m}\right) \neq \emptyset .
$$

Let $t_{n}=\max \left\{t_{n, m}: 1 \leq m \leq r\right\}$ and $s_{n}=\max \left\{s_{n, m}: 1 \leq m \leq r\right\}$. Assume that $\sum_{n=1}^{\infty}\left(k_{n}-1\right)<$ $\infty$, where $k_{n}=\max \left\{s_{n}, t_{n}: 1 \leq m \leq r\right\}$. Let $\left\{x_{n}\right\}_{n=0}^{\infty}$ be a sequence generated by $(\Upsilon)$, where $\left\{\alpha_{n}\right\},\left\{\beta_{n, 1}\right\},\left\{\beta_{n, 2}\right\}, \ldots,\left\{\beta_{n, r}\right\},\left\{\gamma_{n, 1}\right\},\left\{\gamma_{n, 2}\right\}, \ldots,\left\{\gamma_{n, r}\right\},\left\{a_{n}\right\},\left\{b_{n, 1}\right\},\left\{b_{n, 2}\right\}, \ldots,\left\{b_{n, r}\right\},\left\{c_{n, 1}\right\}$, $\left\{c_{n, 2}\right\}, \ldots,\left\{c_{n, r}\right\}$ be real number sequences in $(0,1)$ such that $\alpha_{n}+\sum_{m=1}^{r} \beta_{n, m}+\sum_{m=1}^{r} \gamma_{n, m}=$ $a_{n}+\sum_{m=1}^{r} b_{n, m}+\sum_{m=1}^{r} c_{n, m}=1$. Assume that the following restrictions imposed on the control sequence $\left\{\alpha_{n}\right\},\left\{\beta_{n, 1}\right\},\left\{\beta_{n, 2}\right\}, \ldots,\left\{\beta_{n, r}\right\},\left\{\gamma_{n, 1}\right\},\left\{\gamma_{n, 2}\right\}, \ldots,\left\{\gamma_{n, r}\right\},\left\{a_{n}\right\},\left\{b_{n, 1}\right\},\left\{b_{n, 2}\right\}, \ldots$, $\left\{b_{n, r}\right\},\left\{c_{n, 1}\right\},\left\{c_{n, 2}\right\}, \ldots,\left\{c_{n, r}\right\}$ are satisfied

(a) $\liminf _{n \rightarrow \infty} \alpha_{n} \beta_{n, m}>0, \liminf _{n \rightarrow \infty} \alpha_{n} \gamma_{n, m}>0$ and $\liminf _{n \rightarrow \infty} a_{n} b_{n, m}>0$, $\forall m \in\{1,2, \ldots, r\}$; 
(b) $\sum_{m=1}^{r} \gamma_{n, m} t_{n}\left(a_{n}+\sum_{m=1}^{r} b_{n, m} s_{n}+\sum_{m=1}^{r} c_{n, m} t_{n}\right)<1$.

Then

$$
\lim _{n \rightarrow \infty}\left\|x_{n}-S_{m} x_{n}\right\|=\lim _{n \rightarrow \infty}\left\|x_{n}-T_{m} x_{n}\right\|=0, \quad \forall m \in\{1,2, \ldots, r\} .
$$

Proof Step 1. Taking $p \in \mathcal{F}$, we see that

$$
\begin{aligned}
\left\|x_{n}-p\right\| & \leq \alpha_{n}\left\|x_{n-1}-p\right\|+\sum_{m=1}^{r} \beta_{n, m}\left\|S_{m}^{n} x_{n-1}-p\right\|+\sum_{m=1}^{r} \gamma_{n, m}\left\|T_{m}^{n} y_{n}-p\right\| \\
& \leq\left(\alpha_{n}+\sum_{m=1}^{r} \beta_{n, m} k_{n}\right)\left\|x_{n-1}-p\right\|+\sum_{m=1}^{r} \gamma_{n, m} k_{n}\left\|y_{n}-p\right\|
\end{aligned}
$$

and

$$
\begin{aligned}
\left\|y_{n}-p\right\| & \leq a_{n}\left\|x_{n}-p\right\|+\sum_{m=1}^{r} b_{n, m}\left\|S_{m}^{n} x_{n}-p\right\|+\sum_{m=1}^{r} c_{n, m}\left\|T_{m}^{n} x_{n}-p\right\| \\
& \leq a_{n} k_{n}\left\|x_{n}-p\right\|+\sum_{m=1}^{r} b_{n, m} k_{n}\left\|x_{n}-p\right\|+\sum_{m=1}^{r} c_{n, m} k_{n}\left\|x_{n}-p\right\| \\
& \leq k_{n}\left\|x_{n}-p\right\| .
\end{aligned}
$$

Substituting (3.2) into (3.1), we have

$$
\left\|x_{n}-p\right\| \leq\left(\alpha_{n}+\sum_{m=1}^{r} \beta_{n, m} k_{n}\right)\left\|x_{n-1}-p\right\|+\sum_{m=1}^{r} \gamma_{n, m} k_{n}^{2}\left\|x_{n}-p\right\| .
$$

In view of $\liminf _{n \rightarrow \infty} \alpha_{n} \beta_{n, m}>0$, and $\alpha_{n}+\sum_{m=1}^{r} \beta_{n, m}+\sum_{m=1}^{r} \gamma_{n, m}=1$, we see that there exists some positive integer $n_{1}$, and a real number $h$, where $h \in(0,1)$, such that

$$
\sum_{m=1}^{r} \gamma_{n, m} \leq h, \quad n \geq n_{1}
$$

Since $\sum_{n=1}^{\infty}\left(k_{n}-1\right)<\infty$, we find that there exists some positive integer $n_{2}$ such that $k_{n}^{2} \leq$ $1+\frac{1-h}{2 h}, \forall n \geq n_{2}$. It follows that

$$
\sum_{m=1}^{r} \gamma_{n, m} k_{n}^{2} \leq u<1, \quad \forall n \geq n_{3},
$$

where $u=h\left(1+\frac{1-h}{2 h}\right)$, and $n_{3} \geq \max \left\{n_{1}, n_{2}\right\}$. It follows (3.3) that

$$
\begin{aligned}
\left\|x_{n}-p\right\| & \leq\left(\frac{\alpha_{n}+\sum_{m=1}^{r} \beta_{n, m} k_{n}}{1-\sum_{m=1}^{r} \gamma_{n, m} k_{n}^{2}}\right)\left\|x_{n-1}-p\right\| \\
& \leq\left(1+\frac{\alpha_{n}+\sum_{m=1}^{r} \beta_{n, m} k_{n}+\sum_{m=1}^{r} \gamma_{n, m} k_{n}^{2}-1}{1-\sum_{m=1}^{r} \gamma_{n, m} k_{n}^{2}}\right)\left\|x_{n-1}-p\right\| \\
& \leq\left(1+\frac{k_{n}^{2}-1}{1-u}\right)\left\|x_{n-1}-p\right\| .
\end{aligned}
$$


It follows from Lemma 2.2 that $\lim _{n \rightarrow \infty}\left\|x_{n}-p\right\|$ exists. This implies that the sequence $\left\{x_{n}\right\}$ is bounded.

On the other hand, we find from Lemma 2.3 that

$$
\begin{aligned}
& \left\|x_{n}-p\right\|^{2} \\
& \leq \alpha_{n}\left\|x_{n-1}-p\right\|^{2}+\sum_{m=1}^{r} \beta_{n, m}\left\|S_{m}^{n} x_{n-1}-p\right\|^{2} \\
& \quad+\sum_{m=1}^{r} \gamma_{n, m}\left\|T_{m}^{n} y_{n}-p\right\|^{2}-\alpha_{n} \beta_{n, m} P\left(\left\|x_{n-1}-S_{m}^{n} x_{n-1}\right\|\right) \\
& \leq \quad\left(\alpha_{n}+\sum_{m=1}^{r} \beta_{n, m} k_{n}^{2}\right)\left\|x_{n-1}-p\right\|^{2}+\sum_{m=1}^{r} \gamma_{n, m} k_{n}^{3}\left\|x_{n}-p\right\|^{2} \\
& \quad-\alpha_{n} \beta_{n, m} P\left(\left\|x_{n-1}-S_{m}^{n} x_{n-1}\right\|\right), \quad \forall m \in\{1,2, \ldots, r\} .
\end{aligned}
$$

It implies that

$$
\begin{aligned}
& \alpha_{n} \beta_{n, m} P\left(\left\|x_{n-1}-S_{m}^{n} x_{n-1}\right\|\right) \\
& \leq\left(\alpha_{n} k_{n}+\sum_{m=1}^{r} \beta_{n, m} k_{n}^{2}\right)\left\|x_{n-1}-p\right\|^{2}+\sum_{m=1}^{r} \gamma_{n, m} k_{n}^{3}\left\|x_{n}-p\right\|^{2} \\
&-k_{n}^{3}\left\|x_{n}-p\right\|^{2}+\left(k_{n}^{3}-1\right)\left\|x_{n}-p\right\|^{2} \\
& \leq\left(\alpha_{n} k_{n}^{3}+\sum_{m=1}^{r} \beta_{n, m} k_{n}^{3}\right)\left(\left\|x_{n-1}-p\right\|^{2}-\left\|x_{n}-p\right\|^{2}\right) \\
&+\left(k_{n}^{3}-1\right)\left\|x_{n}-p\right\|^{2}, \quad \forall m \in\{1,2, \ldots, r\} .
\end{aligned}
$$

Since $\lim _{n \rightarrow \infty}\left\|x_{n}-p\right\|$ exists, we find from restriction (a) that

$$
\lim _{n \rightarrow \infty} P\left(\left\|x_{n-1}-S_{m}^{n} x_{n-1}\right\|\right)=0
$$

for every $m \in\{1,2, \ldots, r\}$. It follows that

$$
\lim _{n \rightarrow \infty}\left\|x_{n-1}-S_{m}^{n} x_{n-1}\right\|=0, \quad \forall m \in\{1,2, \ldots, r\}
$$

In view of Lemma 2.3, we have

$$
\begin{aligned}
\left\|x_{n}-p\right\|^{2} \leq & \alpha_{n}\left\|x_{n-1}-p\right\|^{2}+\sum_{m=1}^{r} \beta_{n, m}\left\|S_{m}^{n} x_{n-1}-p\right\|^{2} \\
& +\sum_{m=1}^{r} \gamma_{n, m}\left\|T_{m}^{n} y_{n}-p\right\|^{2}-\alpha_{n} \gamma_{n, m} P\left(\left\|x_{n-1}-T_{m}^{n} y_{n}\right\|\right) \\
\leq & \left(\alpha_{n}+\sum_{m=1}^{r} \beta_{n, m} k_{n}^{2}\right)\left\|x_{n-1}-p\right\|^{2}+\sum_{m=1}^{r} \gamma_{n, m} k_{n}^{3}\left\|x_{n}-p\right\|^{2} \\
& -\alpha_{n} \gamma_{n, m} P\left(\left\|x_{n-1}-T_{m}^{n} y_{n}\right\|\right), \quad \forall m \in\{1,2, \ldots, r\} .
\end{aligned}
$$


It implies that

$$
\begin{aligned}
& \alpha_{n} \gamma_{n, m} P\left(\left\|x_{n-1}-T_{m}^{n} y_{n}\right\|\right) \\
& \leq\left(\alpha_{n} k_{n}+\sum_{m=1}^{r} \beta_{n, m} k_{n}^{2}\right)\left\|x_{n-1}-p\right\|^{2}+\sum_{m=1}^{r} \gamma_{n, m} k_{n}^{3}\left\|x_{n}-p\right\|^{2} \\
&-k_{n}^{3}\left\|x_{n}-p\right\|^{2}+\left(k_{n}^{3}-1\right)\left\|x_{n}-p\right\|^{2} \\
& \leq\left(\alpha_{n} k_{n}^{3}+\sum_{m=1}^{r} \beta_{n, m} k_{n}^{3}\right)\left(\left\|x_{n-1}-p\right\|^{2}-\left\|x_{n}-p\right\|^{2}\right) \\
&+\left(k_{n}^{3}-1\right)\left\|x_{n}-p\right\|^{2}, \quad \forall m \in\{1,2, \ldots, r\} .
\end{aligned}
$$

Since $\lim _{n \rightarrow \infty}\left\|x_{n}-p\right\|$ exists, from the condition (a), we have that

$$
\lim _{n \rightarrow \infty} P\left(\left\|x_{n-1}-T_{m}^{n} y_{n}\right\|\right)=0
$$

for every $m \in\{1,2, \ldots, r\}$. It follows that

$$
\lim _{n \rightarrow \infty}\left\|x_{n-1}-T_{m}^{n} y_{n}\right\|=0, \quad \forall m \in\{1,2, \ldots, r\}
$$

Notice that

$$
\left\|x_{n}-x_{n-1}\right\| \leq \sum_{m=1}^{r} \beta_{n, m}\left\|S_{m}^{n} x_{n-1}-x_{n-1}\right\|+\sum_{m=1}^{r} \gamma_{n, m}\left\|T_{m}^{n} y_{n}-x_{n-1}\right\|
$$

In the light of (3.5), and (3.6), we find that

$$
\lim _{n \rightarrow \infty}\left\|x_{n-1}-x_{n}\right\|=0 .
$$

Step 2. Notice that

$$
\left\|x_{n}-T_{m}^{n} y_{n}\right\| \leq\left\|x_{n}-x_{n-1}\right\|+\left\|x_{n-1}-T_{m}^{n} y_{n}\right\|, \quad \forall m \in\{1,2, \ldots, r\} .
$$

It implies from (3.6), and (3.7) that

$$
\lim _{n \rightarrow \infty}\left\|x_{n}-T_{m}^{n} y_{n}\right\|=0, \quad \forall m \in\{1,2, \ldots, r\} .
$$

On the other hand, we have

$$
\begin{aligned}
\left\|x_{n}-S_{m}^{n} x_{n}\right\| \leq & \left\|x_{n}-x_{n-1}\right\|+\left\|x_{n-1}-S_{m}^{n} x_{n-1}\right\| \\
& +\left\|S_{m}^{n} x_{n-1}-S_{m}^{n} x_{n}\right\|, \quad \forall m \in\{1,2, \ldots, r\} .
\end{aligned}
$$

Since $S_{m}$ is Lipschitz for every $m \in\{1,2, \ldots, r\}$, we see from (3.5) and (3.7) that

$$
\lim _{n \rightarrow \infty}\left\|x_{n}-S_{m}^{n} x_{n}\right\|=0, \quad \forall m \in\{1,2, \ldots, r\}
$$


Step 3. In view of $\liminf _{n \rightarrow \infty} a_{n} b_{n, m}>0$, and

$$
a_{n}+\sum_{m=1}^{r} b_{n, m}+\sum_{m=1}^{r} c_{n, m}=1
$$

we see that there exists some positive integer $n_{4}$, and a real number $h^{\prime}$, where $h^{\prime} \in(0,1)$, such that

$$
\sum_{m=1}^{r} c_{n, m} \leq h^{\prime}, \quad n \geq n_{4} .
$$

Since $\sum_{n=1}^{\infty}\left(k_{n}-1\right)<\infty$, we find that there exists a positive integer $n_{5}$ such that $k_{n} \leq 1+\frac{1-h^{\prime}}{2 h^{\prime}}$, $\forall n \geq n_{5}$. It follows that

$$
\sum_{m=1}^{r} c_{n, m} k_{n} \leq u^{\prime}<1, \quad \forall n \geq n_{6}
$$

where $u^{\prime}=h^{\prime}\left(1+\frac{1-h^{\prime}}{2 h^{\prime}}\right)$, and $n_{6} \geq \max \left\{n_{4}, n_{5}\right\}$. It follows that

$$
\begin{aligned}
\left\|x_{n}-y_{n}\right\| & \leq \sum_{m=1}^{r} b_{n, m}\left\|S_{m}^{n} x_{n}-x_{n}\right\|+\sum_{m=1}^{r} c_{n, m}\left\|T_{m}^{n} x_{n}-x_{n}\right\| \\
& \leq \sum_{m=1}^{r} b_{n, m}\left\|S_{m}^{n} x_{n}-x_{n}\right\|+\sum_{m=1}^{r} c_{n, m}\left\|T_{m}^{n} x_{n}-T_{m}^{n} y_{n}\right\|+\sum_{m=1}^{r} c_{n, m}\left\|T_{m}^{n} y_{n}-x_{n}\right\| \\
& \leq \sum_{m=1}^{r} b_{n, m}\left\|S_{m}^{n} x_{n}-x_{n}\right\|+\sum_{m=1}^{r} c_{n, m} k_{n}\left\|x_{n}-y_{n}\right\|+\sum_{m=1}^{r} c_{n, m}\left\|T_{m}^{n} y_{n}-x_{n}\right\| .
\end{aligned}
$$

This implies that

$$
\begin{aligned}
& \left(1-\sum_{m=1}^{r} c_{n, m} k_{n}\right)\left\|x_{n}-y_{n}\right\| \\
& \leq \sum_{m=1}^{r} b_{n, m}\left\|S_{m}^{n} x_{n}-x_{n}\right\|+\sum_{m=1}^{r} c_{n, m}\left\|T_{m}^{n} y_{n}-x_{n}\right\| .
\end{aligned}
$$

It follows from (3.8) and (3.9) that

$$
\lim _{n \rightarrow \infty}\left\|x_{n}-y_{n}\right\|=0 \text {. }
$$

Step 4. Notice that

$$
\begin{aligned}
\left\|y_{n}-y_{n-1}\right\| \leq & a_{n}\left\|x_{n}-x_{n-1}\right\|+\sum_{m=1}^{r} b_{n, m}\left\|S_{m}^{n} x_{n}-x_{n-1}\right\| \\
& +\sum_{m=1}^{r} c_{n, m}\left\|T_{m}^{n} x_{n}-x_{n-1}\right\|+\left\|x_{n-1}-y_{n-1}\right\| \\
\leq & a_{n}\left\|x_{n}-x_{n-1}\right\|+\sum_{m=1}^{r} b_{n, m}\left\|S_{m}^{n} x_{n}-x_{n}\right\|
\end{aligned}
$$




$$
\begin{aligned}
& +\sum_{m=1}^{r} b_{n, m}\left\|x_{n}-x_{n-1}\right\|+\sum_{m=1}^{r} c_{n, m}\left\|T_{m}^{n} x_{n}-T_{m}^{n} y_{n}\right\| \\
& +\sum_{m=1}^{r} c_{n, m}\left\|T_{m}^{n} y_{n}-x_{n-1}\right\|+\left\|x_{n-1}-y_{n-1}\right\| .
\end{aligned}
$$

Since $T_{m}$ is Lipschitz for every $m \in\{1,2, \ldots, r\}$, we see from (3.6), (3.7), (3.9), and (3.10) that

$$
\lim _{n \rightarrow \infty}\left\|y_{n}-y_{n-1}\right\|=0
$$

Step 5. Notice that

$$
\begin{aligned}
\left\|x_{n}-S_{m} x_{n}\right\| \leq & \left\|x_{n}-x_{n+1}\right\|+\left\|x_{n+1}-S_{m}^{n+1} x_{n+1}\right\| \\
& +\left\|S_{m}^{n+1} x_{n+1}-S_{m}^{n+1} x_{n}\right\|+\left\|S_{m}^{n+1} x_{n}-S_{m} x_{n}\right\| \\
\leq & (1+M)\left\|x_{n}-x_{n+1}\right\|+\left\|x_{n+1}-S_{m}^{n+1} x_{n+1}\right\| \\
& +M\left\|S_{m}^{n} x_{n}-x_{n}\right\|,
\end{aligned}
$$

where $M=\sup _{n \geq 1}\left\{k_{n}\right\}$. It follows from (3.7) and (3.9) that

$$
\lim _{n \rightarrow \infty}\left\|x_{n}-S_{m} x_{n}\right\|=0, \quad \forall m \in\{1,2, \ldots, r\}
$$

On the other hand, we have

$$
\begin{aligned}
\left\|x_{n}-T_{m} x_{n}\right\| \leq & \left\|x_{n}-x_{n+1}\right\|+\left\|x_{n+1}-T_{m}^{n+1} y_{n+1}\right\| \\
& +\left\|T_{m}^{n+1} y_{n+1}-T_{m}^{n+1} y_{n}\right\|+\left\|T_{m}^{n+1} y_{n}-T_{m} x_{n}\right\| \\
\leq & \left\|x_{n}-x_{n+1}\right\|+\left\|x_{n+1}-T_{m}^{n+1} y_{n+1}\right\|+M\left\|y_{n+1}-y_{n}\right\| \\
& +M\left\|T_{m}^{n} y_{n}-x_{n}\right\| .
\end{aligned}
$$

It follows from (3.7), (3.8), and (3.11) that

$$
\lim _{n \rightarrow \infty}\left\|x_{n}-T_{m} x_{n}\right\|=0, \quad \forall m \in\{1,2, \ldots, r\}
$$

This completes the proof.

Next, we give the following weak convergence theorems with the help of Opial's condition.

Theorem 3.2 Let $C$ be a nonempty, closed, and convex subset of a uniformly convex Banach space $E$ with Opial's condition. Let $S_{m}: C \rightarrow C$ be an asymptotically nonexpansive mapping with the sequence $\left\{s_{n, m}\right\}$, and $T_{m}: C \rightarrow C$ an asymptotically nonexpansive mapping with the sequence $\left\{t_{n, m}\right\}$, for every $m \in\{1,2, \ldots, r\}$, where $r \geq 1$ is some positive integer. Assume that

$$
\mathcal{F}=\bigcap_{m=1}^{r} F\left(S_{m}\right) \cap \bigcap_{m=1}^{r} F\left(T_{m}\right) \neq \emptyset .
$$


Let $t_{n}=\max \left\{t_{n, m}: 1 \leq m \leq r\right\}$, and $s_{n}=\max \left\{s_{n, m}: 1 \leq m \leq r\right\}$. Assume that $\sum_{n=1}^{\infty}\left(k_{n}-1\right)<$ $\infty$, where $k_{n}=\max \left\{s_{n}, t_{n}: 1 \leq m \leq r\right\}$. Let $\left\{x_{n}\right\}_{n=0}^{\infty}$ be a sequence generated by $(\Upsilon)$, where $\left\{\alpha_{n}\right\},\left\{\beta_{n, 1}\right\},\left\{\beta_{n, 2}\right\}, \ldots,\left\{\beta_{n, r}\right\},\left\{\gamma_{n, 1}\right\},\left\{\gamma_{n, 2}\right\}, \ldots$, and $\left\{\gamma_{n, r}\right\},\left\{a_{n}\right\},\left\{b_{n, 1}\right\},\left\{b_{n, 2}\right\}, \ldots,\left\{b_{n, r}\right\}$, $\left\{c_{n, 1}\right\},\left\{c_{n, 2}\right\}, \ldots$, and $\left\{c_{n, r}\right\}$ be real number sequences in $(0,1)$ such that

$$
\alpha_{n}+\sum_{m=1}^{r} \beta_{n, m}+\sum_{m=1}^{r} \gamma_{n, m}=a_{n}+\sum_{m=1}^{r} b_{n, m}+\sum_{m=1}^{r} c_{n, m}=1 .
$$

Assume that restrictions (a) and (b) as in Theorem 3.1 are satisfied. Then $\left\{x_{n}\right\}$ converges weakly to some point in $\mathcal{F}$.

Proof Since $\left\{x_{n}\right\}$ is bounded, there exists a subsequence $\left\{x_{n_{i}}\right\} \subset\left\{x_{n}\right\}$ such that $\left\{x_{n_{i}}\right\}$ converges weakly to a point $\bar{x} \in C$. It follows from Lemma 2.1 that $\bar{x} \in \mathcal{F}$. Assume that there exists another subsequence $\left\{x_{n_{j}}\right\} \subset\left\{x_{n}\right\}$ such that $\left\{x_{n_{j}}\right\}$ converges weakly to a point $\hat{x} \in C$. It follows from Lemma 2.1 that $\hat{x} \in \mathcal{F}$. If $\bar{x} \neq \hat{x}$, then

$$
\begin{aligned}
\lim _{n \rightarrow \infty}\left\|x_{n}-\bar{x}\right\| & =\liminf _{i \rightarrow \infty}\left\|x_{n_{i}}-\bar{x}\right\|<\liminf _{i \rightarrow \infty}\left\|x_{n_{i}}-\hat{x}\right\| \\
& =\liminf _{j \rightarrow \infty}\left\|x_{n_{j}}-\hat{x}\right\|<\liminf _{j \rightarrow \infty}\left\|x_{n_{j}}-\bar{x}\right\| \\
& =\lim _{n \rightarrow \infty}\left\|x_{n}-\bar{x}\right\| .
\end{aligned}
$$

This is a contradiction. Hence, $\bar{x}=\hat{x}$. This completes the proof.

If $r=1$, then Theorem 3.2 is reduced to the following.

Corollary 3.1 Let $C$ be a nonempty, closed, and convex subset of a uniformly convex Banach space $E$ with Opial's condition. Let $S: C \rightarrow C$ be an asymptotically nonexpansive mapping with the sequence $\left\{s_{n}\right\}$, and $T: C \rightarrow C$ an asymptotically nonexpansive mapping with the sequence $\left\{t_{n}\right\}$. Assume that

$$
\mathcal{F}=F(S) \cap F(T) \neq \emptyset \text {. }
$$

Assume that $\sum_{n=1}^{\infty}\left(k_{n}-1\right)<\infty$, where $k_{n}=\max \left\{s_{n}, t_{n}: 1 \leq m \leq r\right\}$. Let $\left\{x_{n}\right\}_{n=0}^{\infty}$ be a sequence generated by the following:

$$
\left\{\begin{array}{l}
x_{0} \in C, \\
x_{n}=\alpha_{n} x_{n-1}+\beta_{n} S^{n} x_{n-1}+\gamma_{n} T^{n} y_{n}, \\
y_{n}=a_{n} x_{n}+b_{n} S^{n} x_{n}+c_{n} T^{n} x_{n}, \quad \forall n \geq 1 .
\end{array}\right.
$$

where $\left\{\alpha_{n}\right\},\left\{\beta_{n}\right\},\left\{\gamma_{n}\right\},\left\{a_{n}\right\},\left\{b_{n}\right\}$, and $\left\{c_{n}\right\}$ are real number sequences in $(0,1)$ such that $\alpha_{n}+\beta_{n}+\gamma_{n}=a_{n}+b_{n}+c_{n}=1$. Assume that the following restrictions imposed on the control sequences $\left\{\alpha_{n}\right\},\left\{\beta_{n}\right\},\left\{\gamma_{n}\right\},\left\{a_{n}\right\},\left\{b_{n}\right\}$, and $\left\{c_{n}\right\}$ are satisfied:

(a) $\liminf _{n \rightarrow \infty} \alpha_{n} \beta_{n}>0, \liminf _{n \rightarrow \infty} \alpha_{n} \gamma_{n}>0$ and $\liminf _{n \rightarrow \infty} a_{n} b_{n}>0$;

(b) $\gamma_{n} t_{n}\left(a_{n}+b_{n} s_{n}+c_{n} t_{n}\right)<1$.

Then $\left\{x_{n}\right\}$ converges weakly to some point in $\mathcal{F}$.

If $b_{n, m}=c_{n, m}=0$, than Theorem 3.2 reduced the following. 
Corollary 3.2 Let $C$ be a nonempty, closed, and convex subset of a uniformly convex Banach space $E$ with Opial's condition. Let $S_{m}: C \rightarrow C$ be an asymptotically nonexpansive mapping with the sequence $\left\{s_{n, m}\right\}$, and $T_{m}: C \rightarrow C$ an asymptotically nonexpansive mapping with the sequence $\left\{t_{n, m}\right\}$, for every $m \in\{1,2, \ldots, r\}$. where $r \geq 1$ is some positive integer. Assume that

$$
\mathcal{F}=\bigcap_{m=1}^{r} F\left(S_{m}\right) \cap \bigcap_{m=1}^{r} F\left(T_{m}\right) \neq \emptyset .
$$

Let $t_{n}=\max \left\{t_{n, m}: 1 \leq m \leq r\right\}$, and $s_{n}=\max \left\{s_{n, m}: 1 \leq m \leq r\right\}$. Assume that $\sum_{n=1}^{\infty}\left(k_{n}-1\right)<$ $\infty$, where $k_{n}=\max \left\{s_{n}, t_{n}: 1 \leq m \leq r\right\}$. Let $\left\{x_{n}\right\}_{n=0}^{\infty}$ be a sequence generated by the following:

$$
x_{0} \in C, \quad x_{n}=\alpha_{n} x_{n-1}+\sum_{m=1}^{r} \beta_{n, m} S_{m}^{n} x_{n-1}+\sum_{m=1}^{r} \gamma_{n, m} T_{m}^{n} x_{n}, \quad \forall n \geq 1
$$

where $\left\{\alpha_{n}\right\},\left\{\beta_{n, 1}\right\},\left\{\beta_{n, 2}\right\}, \ldots,\left\{\beta_{n, r}\right\},\left\{\gamma_{n, 1}\right\},\left\{\gamma_{n, 2}\right\}, \ldots$, and $\left\{\gamma_{n, r}\right\}$, are real number sequences in $(0,1)$ such that $\alpha_{n}+\sum_{m=1}^{r} \beta_{n, m}+\sum_{m=1}^{r} \gamma_{n, m}=1$. Assume that the following restrictions imposed on the control sequences $\left\{\alpha_{n}\right\},\left\{\beta_{n, 1}\right\},\left\{\beta_{n, 2}\right\}, \ldots,\left\{\beta_{n, r}\right\},\left\{\gamma_{n, 1}\right\},\left\{\gamma_{n, 2}\right\}, \ldots$, and $\left\{\gamma_{n, r}\right\}$ are satisfied

(a) $\liminf _{n \rightarrow \infty} \alpha_{n} \beta_{n, m}>0$, and $\liminf _{n \rightarrow \infty} \alpha_{n} \gamma_{n, m}>0, \forall m \in\{1,2, \ldots r\}$;

(b) $\sum_{m=1}^{r} \gamma_{n, m} t_{n}<1$.

Then $\left\{x_{n}\right\}$ converges weakly to some point in $\mathcal{F}$.

If $\beta_{n, m}=b_{n, m}=0$, than Theorem 3.2 reduced the following.

Corollary 3.3 Let $C$ be a nonempty, closed, and convex subset of a uniformly convex $B a$ nach space $E$ with Opial's condition. Let $T_{m}: C \rightarrow C$ be an asymptotically nonexpansive mapping with the sequence $\left\{t_{n, m}\right\}$, for every $m \in\{1,2, \ldots, r\}$, where $r \geq 1$ is some positive integer. Assume that

$$
\mathcal{F}=\bigcap_{m=1}^{r} F\left(T_{m}\right) \neq \emptyset .
$$

Assume that $\sum_{n=1}^{\infty}\left(t_{n}-1\right)<\infty$, where $t_{n}=\max \left\{t_{n, m}: 1 \leq m \leq r\right\}$. Let $\left\{x_{n}\right\}_{n=0}^{\infty}$ be a sequence generated by the following:

$$
\left\{\begin{array}{l}
x_{0} \in C, \\
x_{n}=\alpha_{n} x_{n-1}+\sum_{m=1}^{r} \gamma_{n, m} T_{m}^{n} y_{n}, \\
y_{n}=a_{n} x_{n}+\sum_{m=1}^{r} c_{n, m} T_{m}^{n} x_{n}, \quad \forall n \geq 1,
\end{array}\right.
$$

where $\left\{\alpha_{n}\right\},\left\{\gamma_{n, 1}\right\},\left\{\gamma_{n, 2}\right\}, \ldots,\left\{\gamma_{n, r}\right\},\left\{a_{n}\right\},\left\{c_{n, 1}\right\},\left\{c_{n, 2}\right\}, \ldots,\left\{c_{n, r}\right\}$ be real number sequences in $(0,1)$ such that

$$
\alpha_{n}+\sum_{m=1}^{r} \gamma_{n, m}=a_{n}+\sum_{m=1}^{r} c_{n, m}=1
$$

Assume that the following restrictions imposed on the control sequence $\left\{\alpha_{n}\right\},\left\{\gamma_{n, 1}\right\},\left\{\gamma_{n, 2}\right\}$, $\ldots,\left\{\gamma_{n, r}\right\},\left\{a_{n}\right\},\left\{c_{n, 1}\right\},\left\{c_{n, 2}\right\}, \ldots,\left\{c_{n, r}\right\}$ are satisfied 
(a) $\liminf _{n \rightarrow \infty} \alpha_{n}>0, \liminf _{n \rightarrow \infty} \alpha_{n} \gamma_{n, m}>0$ and $\liminf _{n \rightarrow \infty} a_{n}>0, \forall m \in\{1,2, \ldots, r\}$;

(b) $\sum_{m=1}^{r} \gamma_{n, m} t_{n}\left(a_{n}+\sum_{m=1}^{r} c_{n, m} t_{n}\right)<1$.

Then $\left\{x_{n}\right\}$ converges weakly to some point in $\mathcal{F}$.

If $S_{m}=I$, where $I$ stands for the identity mappings, then Theorem 3.2 reduced the following.

Corollary 3.4 Let $C$ be a nonempty, closed, and convex subset of a uniformly convex Banach space $E$ with Opial's condition. Let $T_{m}: C \rightarrow C$ be an asymptotically nonexpansive mapping with the sequence $\left\{t_{n, m}\right\}$, for every $m \in\{1,2, \ldots, r\}$, where $r \geq 1$ is some positive integer. Assume that

$$
\mathcal{F}=\bigcap_{m=1}^{r} F\left(T_{m}\right) \neq \emptyset .
$$

Asume that $\sum_{n=1}^{\infty}\left(t_{n}-1\right)<\infty$, where $t_{n}=\max \left\{t_{n, m}: 1 \leq m \leq r\right\}$. Let $\left\{x_{n}\right\}_{n=0}^{\infty}$ be a sequence generated by the following:

$$
\left\{\begin{array}{l}
x_{0} \in C, \\
x_{n}=\left(\alpha_{n}+\sum_{m=1}^{r} \beta_{n, m}\right) x_{n-1}+\sum_{m=1}^{r} \gamma_{n, m} T_{m}^{n} y_{n}, \\
y_{n}=\left(a_{n}+\sum_{m=1}^{r} b_{n, m}\right) x_{n}+\sum_{m=1}^{r} c_{n, m} T_{m}^{n} x_{n}, \quad \forall n \geq 1,
\end{array}\right.
$$

where $\left\{\alpha_{n}\right\},\left\{\beta_{n, 1}\right\},\left\{\beta_{n, 2}\right\}, \ldots,\left\{\beta_{n, r}\right\},\left\{\gamma_{n, 1}\right\},\left\{\gamma_{n, 2}\right\}, \ldots,\left\{\gamma_{n, r}\right\},\left\{a_{n}\right\},\left\{b_{n, 1}\right\},\left\{b_{n, 2}\right\}, \ldots,\left\{b_{n, r}\right\},\left\{c_{n, 1}\right\}$, $\left\{c_{n, 2}\right\}, \ldots,\left\{c_{n, r}\right\}$ be real number sequences in $(0,1)$ such that $\alpha_{n}+\sum_{m=1}^{r} \beta_{n, m}+\sum_{m=1}^{r} \gamma_{n, m}=$ $a_{n}+\sum_{m=1}^{r} b_{n, m}+\sum_{m=1}^{r} c_{n, m}=1$. Assume that the following restrictions imposed on the control sequences $\left\{\alpha_{n}\right\},\left\{\beta_{n, 1}\right\},\left\{\beta_{n, 2}\right\}, \ldots,\left\{\beta_{n, r}\right\},\left\{\gamma_{n, 1}\right\},\left\{\gamma_{n, 2}\right\}, \ldots,\left\{\gamma_{n, r}\right\},\left\{a_{n}\right\},\left\{b_{n, 1}\right\},\left\{b_{n, 2}\right\}, \ldots$, $\left\{b_{n, r}\right\},\left\{c_{n, 1}\right\},\left\{c_{n, 2}\right\}, \ldots,\left\{c_{n, r}\right\}$ are satisfied

(a) $\liminf _{n \rightarrow \infty} \alpha_{n} \beta_{n, m}>0, \liminf _{n \rightarrow \infty} \alpha_{n} \gamma_{n, m}>0$ and $\liminf _{n \rightarrow \infty} a_{n} b_{n, m}>0$, $\forall m \in\{1,2, \ldots, r\}$

(b) $\sum_{m=1}^{r} \gamma_{n, m} t_{n}\left(a_{n}+\sum_{m=1}^{r} b_{n, m}+\sum_{m=1}^{r} c_{n, m} t_{n}\right)<1$.

Then $\left\{x_{n}\right\}$ converges weakly to some point in $\mathcal{F}$.

If $T_{m}=I, b_{n, m}=0$, where $I$ stands for the identity mappings, then Theorem 3.2 reduced the following.

Corollary 3.5 Let $C$ be a nonempty, closed, and convex subset of a uniformly convex Banach spaces $E$ with Opial's condition. Let $S_{m}: C \rightarrow C$ be an asymptotically nonexpansive mapping with the sequence $\left\{s_{n, m}\right\}$, for every $m \in\{1,2, \ldots, r\}$, where $r \geq 1$ is some positive integer. Assume that

$$
\mathcal{F}=\bigcap_{m=1}^{r} F\left(S_{m}\right) \neq \emptyset .
$$


Assume that $\sum_{n=1}^{\infty}\left(s_{n}-1\right)<\infty$, where $s_{n}=\max \left\{s_{n, m}: 1 \leq m \leq r\right\}$. Let $\left\{x_{n}\right\}_{n=0}^{\infty}$ be a sequence generated by the following:

$$
\left\{\begin{array}{l}
x_{0} \in C, \\
x_{n}=\frac{\alpha_{n}}{1-\sum_{m=1}^{r}\left(a_{n}+\sum_{m=1}^{r} c_{n, m}\right)} x_{n-1}+\frac{\sum_{m=1}^{r} \beta_{n, m} S_{m}^{n}}{1-\sum_{m=1}^{r}\left(a_{n}+\sum_{m=1}^{r} c_{n, m}\right)} x_{n-1}, \quad \forall n \geq 1,
\end{array}\right.
$$

where $\left\{\alpha_{n}\right\},\left\{\beta_{n, 1}\right\},\left\{\beta_{n, 2}\right\}, \ldots,\left\{\beta_{n, r}\right\},\left\{\gamma_{n, 1}\right\},\left\{\gamma_{n, 2}\right\}, \ldots,\left\{\gamma_{n, r}\right\},\left\{a_{n}\right\},\left\{c_{n, 1}\right\},\left\{c_{n, 2}\right\}, \ldots,\left\{c_{n, r}\right\}$ be real number sequences in $(0,1)$ such that

$$
\alpha_{n}+\sum_{m=1}^{r} \beta_{n, m}+\sum_{m=1}^{r} \gamma_{n, m}=a_{n}+\sum_{m=1}^{r} c_{n, m}=1
$$

Assume that the following restrictions imposed on the control sequences $\left\{\alpha_{n}\right\},\left\{\beta_{n, 1}\right\},\left\{\beta_{n, 2}\right\}$, $\ldots,\left\{\beta_{n, r}\right\},\left\{\gamma_{n, 1}\right\},\left\{\gamma_{n, 2}\right\}, \ldots,\left\{\gamma_{n, r}\right\},\left\{a_{n}\right\},\left\{c_{n, 1}\right\},\left\{c_{n, 2}\right\}, \ldots,\left\{c_{n, r}\right\}$ are satisfied $\liminf _{n \rightarrow \infty} \alpha_{n} \beta_{n, m}>$ 0 and $\liminf _{n \rightarrow \infty} \alpha_{n} \gamma_{n, m}>0$ for all $m \in\{1,2, \ldots, r\}$. Then $\left\{x_{n}\right\}$ converges weakly to some point in $\mathcal{F}$.

\section{Competing interests}

The authors declare that they have no competing interests.

\section{Authors' contributions}

The main idea of this paper is proposed by JKK. JKK and WHL prepared the manuscript initially and performed all the steps of proof in this research. All authors read and approved the final manuscript.

\section{Acknowledgements}

The research was supported by Kyungnam University Research Fund, 2012.

Received: 28 November 2012 Accepted: 3 April 2013 Published: 16 April 2013

\section{References}

1. Bauschke, HH, Borwein, JM: On projection algorithms for solving convex feasibility problems. SIAM Rev. 38, 367-426 (1996)

2. Kotzer, T, Cohen, N, Shamir, J: Image restoration by a novel method of parallel projection onto constraint sets. Optim. Lett. 20, 1772-1774 (1995)

3. Byrne, C: A unified treatment of some iterative algorithms in signal processing and image reconstruction. Inverse Probl. 20, 103-120 (2008)

4. Censor, Y, Elfving, T, Kopf, N, Bortfeld, T: The multiple-sets split feasibility problem and its applications for inverse problems. Inverse Probl. 21, 2071-2084 (2005)

5. Censor, Y, Bortfeld, T, Martin, B, Trofimov, A: A unified approach for inversion problems in intensity-modulated radiation therapy. Phys. Med. Biol. 51, 2353-2365 (2006)

6. Lopez, G, Martin, V, Xu, HK: Perturbation techniques for nonexpansive mappings with applications. Nonlinear Anal. 10, 2369-2383 (2009)

7. Reich, S: Weak convergence theorems for nonexpansive mappings in Banach spaces. J. Math. Anal. Appl. 67, 274-276 (1979)

8. Schu, J: Weak and strong convergence to fixed points of asymptotically nonexpansive mappings. Bull. Aust. Math. Soc. 43, 153-159 (1991)

9. Khan, SH, Yildirim, I, Ozdemir, M: Convergence of an implicit algorithm for two families of nonexpansive mappings. Comput. Math. Appl. 59, 3084-3091 (2010)

10. Acedo, GL, Xu, HK: Iterative methods for strict pseudo-contractions in Hilbert spaces. Nonlinear Anal. 67, 2258-2271 (2007)

11. Qin, X, Kang, SM, Agarwal, RP: On the convergence of an implicit iterative process for generalized asymptotically quasi-nonexpansive mappings. Fixed Point Theory Appl. 2010, Article ID 714860 (2010)

12. Qin, X, Kim, JK, Wang, TZ: On the convergence of implicit iterative processes for asymptotically pseudocontractive mappings in the intermediate sense. Abstr. Appl. Anal. 2011, Article ID 468716 (2011)

13. Qin, X, Cho, SY: Implicit iterative algorithms for treating strongly continuous semigroups of Lipschitz pseudocontractions. Appl. Math. Lett. 23, 1252-1255 (2010)

14. Kim, JK, Nam, YM, Sim, JY: Convergence theorems of implicit iterative sequences for a finite family of asymptotically quasi-nonexpansive type mappings. Nonlinear Anal. 71, e2839-e2848 (2009)

15. Hao, Y, Cho, SY, Qin, X: Some weak convergence theorems for a family of asymptotically nonexpansive nonself mappings. Fixed Point Theory Appl. 2010, Article ID 218573 (2010) 
16. Chang, SS, Tan, KK, Lee, HWJ, Chan, CK: On the convergence of implicit iteration process with error for a finite family of asymptotically nonexpansive mappings. J. Math. Anal. Appl. 313, 273-283 (2006)

17. Tian, YK, Chang, SS, Huang, JL, Wang, X, Kim, JK: Implicit iteration process for common fixed point of strictlt asymptotically pseudocontractive mappings in Banach spaces. Fixed Point Theory Appl. 2008, Article ID 324575 (2008)

18. Opial, Z: Weak convergence of the sequence of successive approximations for nonexpansive mappings. Bull. Am. Math. Soc. 73, 591-597 (1967)

19. Goebel, K, Kirk, WA: A fixed point theorem for asymptotically nonexpansive mappings. Proc. Am. Math. Soc. 35, 171-174 (1972)

20. Chang, SS, Cho, YJ, Zhou, HY: Demi-closed principle and weak convergence problems for asymptotically nonexpansive mappings. J. Korean Math. Soc. 38, 1245-1260 (2001)

21. Tan, KK, Xu, HK: Approximating fixed points of nonexpansive mappings by the Ishikawa iteration process. J. Math. Anal. Appl. 178, 301-308 (1993)

doi:10.1186/1029-242X-2013-179

Cite this article as: Kim and Lim: Convergence theorems of a new iteration for asymptotically nonexpansive

mappings in Banach spaces. Journal of Inequalities and Applications 2013 2013:179.

\section{Submit your manuscript to a SpringerOpen ${ }^{\odot}$ journal and benefit from:}

- Convenient online submission

- Rigorous peer review

- Immediate publication on acceptance

- Open access: articles freely available online

- High visibility within the field

- Retaining the copyright to your article 